\title{
Wireless Communication Algorithm for Mobile Electronic Commerce
}

\author{
Wencan Chen ${ }^{1}$, Xiaodong Liü $^{2}$ a \\ ${ }^{1}$ College of Information Science and Engineering, Ocean University of China, Qingdao 266100, \\ P.R.China \\ ${ }^{2}$ College of Mathematical Sciences, Ocean University of China, Qingdao 266100, P.R.China \\ ${ }^{a}$ liuxiaodong.ouc@hotmail.com
}

Keywords: Wireless communication; Mobile electronic commerce; Transmission Control.

\begin{abstract}
Mobile e-business, with a full use of its mobility, makes information exchange and commercial transaction anytime anywhere become possible. But in the transmission technology and terminal handling ability, there is a huge gap to the traditional e-commerce. According to the existing wireless network communication technology, this paper introduces a new communication control algorithm to the mobile e-commerce transmission control. This algorithm can adjust data sending time according to the user's needs and the constant change in the wireless communication and channels status. In this way, it can reduce the number of redundant data and network energy consumption, and makes the fusion between network and technique under the time requirements become possible.
\end{abstract}

\section{Introduction}

The spread of the Internet has driven and promoted the development of e-commerce. E-commerce makes it possible to complete the transaction all through the Internet, thus changed the traditional face-to-face trading pattern, compared to which E-commerce has such advantages: (1) Reduce the manpower and material resources input and the transaction cost; (2) Greatly simplify the deal flow, improving the transaction efficiency;(3) Break the limitation of time and space ,bringing more business opportunities. E-commerce has dramatically changed the business model and has driven the economic structure transformation. In many countries, the development of e-commerce is unprecedentedly prosperous and transactions through the internet has become a trend.

Along with the development of mobile communication technology, e-commerce gradually expands from wired network to wireless networks, and forms the mobile e-commerce. It combines mobile technology and e-commerce, taking SMS, MMS, WAP and other mobile technology into the application with mobile terminals such as mobile phones and handheld computers to complete online transaction. Along with the increasing number of mobile phone users, mobile e-commerce will become one of the most widely used terminal in the future e-commerce applications. It is reported that by December 31, 2007, the number of mobile phone users in China has reached 5040 million, while the number of domestic mobile phone users has exceeded 600 million, by 21 st China Internet Report released by CNNIC. Meanwhile, as the $3 \mathrm{G}$ is applied in China in a large scale, mobile e-commerce market prospect is very broad. According to the research data offered by British Data-monitor company, the number of global mobile commerce users will achieve 16.7 million in 2008. The annual income will reach 5540 billion dollars. Mobile e-commerce will take up 15\% share of global online trading market.

At present, the differences between mobile e-business and traditional e-commerce difference is mainly manifested in the terminal and network which it carry on .Mobile e-commerce uses mobile phones, PDA and other mobile terminals to trade, while e-commerce generally uses the PC to trade. The loading network of mobile e-commerce is mobile communication. The loading network of e-commerce is the internet. Therefore, from a technical point of view, it's hard for wireless network environment to directly use communication protocol which wired network environment uses. 
Firstly, the compute environment for mobile terminals is very limited. Except for a few expensive Smartphone, most of the mobile phones in the market have many limitations. Compared to PC, mobile terminal which is responsible for processing transactions, is only armed with low-end processors, a small amount of memory ,so it can not handle a large number of trade information and high-complexity operations like PC does. Moreover, the overly complex operations will consume more time resource to carry out transaction processing, causing a delay in providing transaction information to users in time, which will lower the use intention. In addition, limited by the size and power consumption, the display and keyboard are both small.

Secondly, compared with the wired communication network, the mobile communication network resources are also very limited. Most of the mobile communication network's frequency bandwidth is limited, and the data transfer rate is relatively low. For example, the data transfer rate of GSM network is $9.6 \mathrm{kbps}$, and the data transfer rate of $3 \mathrm{G}$ network is up to $384 \mathrm{kbPs}$ or ZMbPs, while the data rate of wired network has reached $100-1000 \mathrm{MbP}$ long before. In addition, the mobile communication network has such deficiencies as a heavy delay, poor reliability of communication links, and high error rate.

It can be concluded that Mobile e-commerce and traditional commerce are different in the Transmission technology and terminal handling ability. Therefore it is improper to directly move the traditional e-commerce technology into the wireless environment. In limited compute environment and communication environment, in order to ensure Communication effect mobile e-commerce, it is necessary to design a compatible solution according to its characteristic.

The WCS given by this article can adaptively adjust sending time according to the user needs and the constant change in the wireless communication and channels status, thus ensure that data is transferred in time to the full extent.

\section{Algorithm Design}

In this section we will introduce the detailed design of WCS algorithm firstly, according to the characteristics of wireless mobile network, we assume that it is possible for the network to appear topology changes. Node sends and receives data in fixed frequency every time.

\section{Algorithm Structure}

Detailed structure of the algorithm as shown in fig. 1.

First of all, as in the document [1] mentioned, recovery unit can make the processed data restored to metadata. At the same time, this unit can separate things which data packet carries such as current channel state, child nodes situation and other information. According to this information ,time control unit can calculate the debug time under the current state.

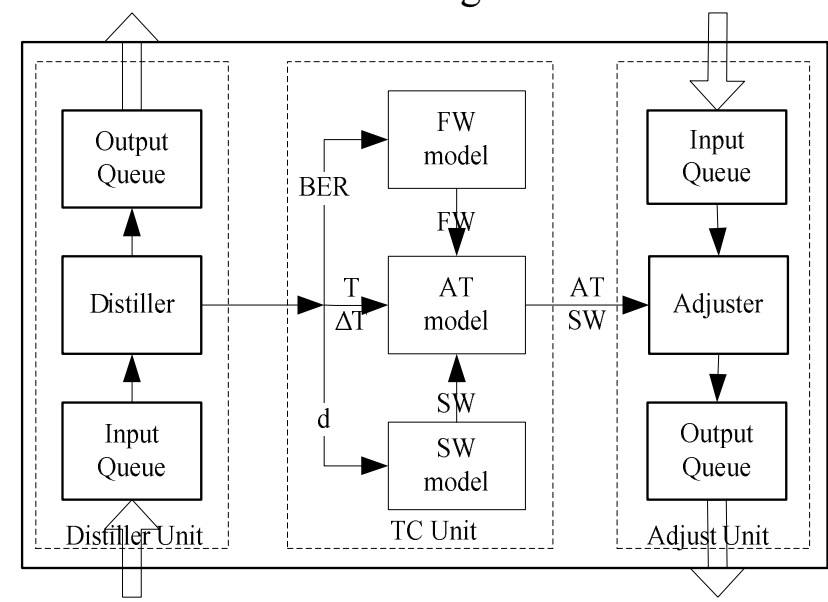

Fig. 1 Algorithm Structure.

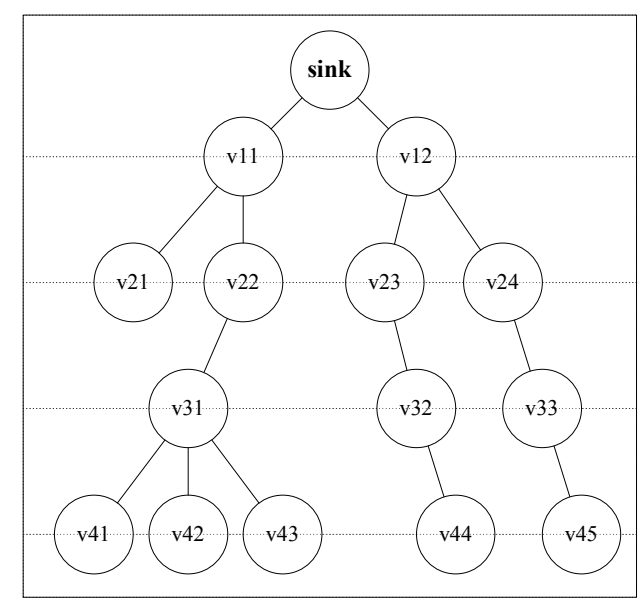

Fig. 2 Schematic diagram of the network 
As the document [1] mentioned, recovery unit and adjustment unit assure the algorithm's modularity, and ensure the enforceability of some related data processing methods such as data fusion and other method.

Here we focus on the core of the algorithm --the design of time control module.

Make $v_{i j}$ represents the network nodes, make $\mathrm{i}$ represents the layer where the node in, make $\mathrm{j}$ represents the sequence number in the same layer. Make $V=\left\{v_{i j}\right\}$ 。 The network structure as shown in Fig. 2.

In this paper we make D represents length of data item, make H represents the length of header, make PER represents the misstatement rate of sending datagram.

Make $S\left(v_{i_{0} j_{0}}\right)=\left\{v_{i j} \mid v_{i j} \in V, i=i_{0}+1, j \in J_{i_{0} j_{0}}\right\}$ represents the collection that composed by each child node of node $v_{i_{0} j_{0}}$ in network. $J_{i_{0} j_{0}}$ presents the corresponding labeling sets. For rigorous reasoning to the article, here we assume that $S\left(v_{i_{0} j_{0}}\right)$ is possible to change. We make degree $\left(v_{i_{0} j_{0}}\right)$ represents the bytes points belong to node $v_{i_{0} j_{0}}$, called the degree of node $v_{i_{0} j_{0}}$. Clearly, degree $\left(v_{i_{0} j_{0}}\right)$ is also possible to change.

For each node, the relationship between false alarm rate $P E R$ and bit error rate $B E R$ is: $P E R=1-(1-B E R)^{(H+D)}$. Thus we define node weight as follows: $w\left(v_{i j}\right)=\frac{1}{(1-B E R)^{(H+D)}}$.

Obviously the weight $w\left(v_{i j}\right)$ is the average of the datagram that required to send a $D$-bit data successfully.Accordingly we define the first weight as follows:

Definition 1: Set arbitrary nodes in the network as $v_{i_{0} j_{0}}$, define the first weight (FW) of that node is the sum of its own weight and the maximum of the first weight of all child nodes : $f w\left(v_{i_{0} j_{0}}\right)=\max _{j \in J_{i_{0} j 0}}\left\{f w\left(v_{i_{0}+1, j}\right)\right\}+w\left(v_{i_{0} j_{0}}\right)$

The first weight of leaf node is its own weight, namely: $f w\left(v_{\text {depth,j }}\right)=w\left(v_{\text {depth,j }}\right)$

Thus, the first weight algorithm as shown in fig.3.

\section{Adjustable Time Calculate Module (AT Module)}

From previous discussions, we know that wireless mobile network should adjust the maximum transmission delay strictly, as required. In this section, we focuse on analyzing how the AT module to calculate the adjustment time according to user's different requirement.

Make $L_{1}$ represents the sub-tree whose root node is node $v_{1, j}$. When the transfer restrictions are given by the user (denoted as T), in order to gather nodes, firstly it should calculate the upper limit of each sub-tree's return time: $\Delta T\left(L_{j}\right)=T-\frac{s w\left(v_{1 j}\right)}{p}, p$ represents the node send frequency.

The adjustment time of root node $v_{1, j}$ is: $\operatorname{at}\left(v_{1, j}\right)=\Delta T\left(L_{j}\right)+\frac{f w\left(v_{1, j}\right)}{p}$

Clearly, $T$ is the most critical of the many constraints which $\Delta T\left(L_{j}\right)$ and $w t\left(v_{1, j}\right)$ suffered.

After get the upper limit of transmission, GET-TIME package which contains the information of $\Delta T\left(L_{j}\right)$ and $s w\left(v_{1, j}\right)$ will be sent to each node of sub-tree $L_{j}$. And each node of sub-tree can get its own transmission time through the equation: $\operatorname{at}\left(v_{i k}\right)=\frac{s w\left(v_{i k}\right)}{s w\left(v_{1 j}\right)} \times \Delta T\left(L_{j}\right)+\frac{f w\left(v_{i k}\right)}{p}$. 


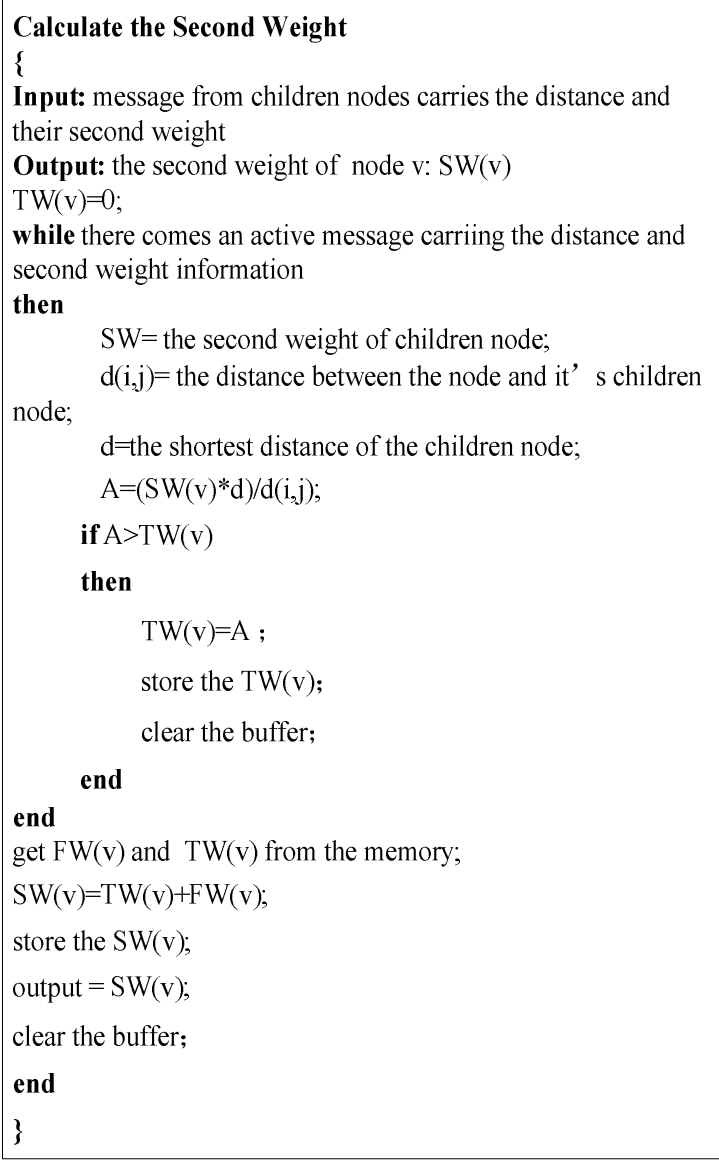

Fig.3 FW Algorithm

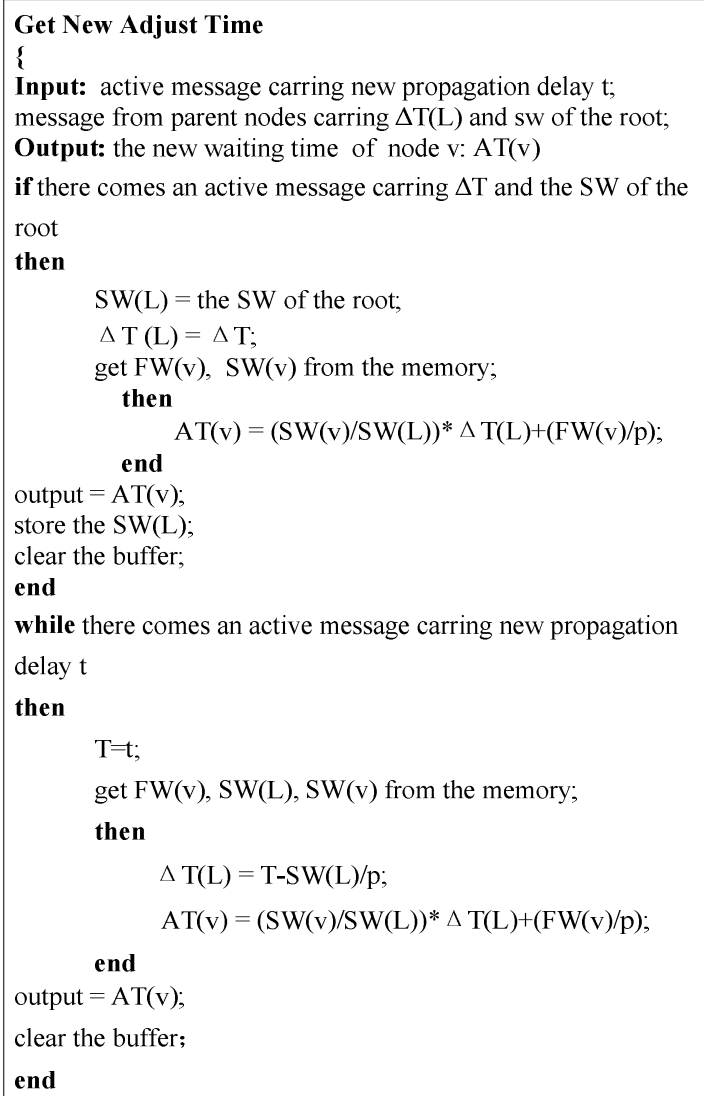

Fig.4 AT Algorithm

After the node receives the message from GET-TIME, it will calculate the waiting time by the algorithm as shown in fig.4, according to the information stored in the FW. From equations given above, we know that because of the difference among BER, FW, and the node degrees, the situation of the conflict between adjacent nodes and channel congestion can be effectively alleviate.

\section{Summary}

This paper introduces a new communications control algorithm in the mobile e-commerce transmission, according to the characteristics of network communication in the Wireless e-commerce, and combining with the communication technology of existing wireless network. According to customer's needs and combining with the changing network topology structure network performance, and other situation, this algorithm can adaptively adjust data sending time. So that it can reduce a lot of redundant data and the network energy, and ensure that the network fusion technology become possible under the time requirement. While the next, as the perfection and testing of the system, we will be on the performance of the algorithm for the further testing.

\section{Acknowledgements}

This project is funded by the Qingdao Scientific and Technological development plan under Grant No. 10-3-4-1-4-jch and by the National Natural Science Foundation of China under Grant No. 60970130 . 


\section{References}

[1] He Tian, Blum Brian M, Stankovic John A.,Abdelzaher T. AIDA: Adaptive Application Independent Aggregation in Sensor Networks. ACM Trans on Embedded Computing System (2003), p.426-457.

[2] Raghunathan V.,Schurgers C.,Park S.,et. Energy Aware Wireless Microsensor Networks. IEEE Signal Processing Magazine (2002), p.40-50.

[3] Heinzelman W., Chandrakasan A., Balakrishnan H., Energy-Efficient Communication Protocol for Wireless Microsensor Networks, the 33Annual Hawaii International Conference on System Sciences. Hawaii (2003), p.01-10.

[4] ZENG peng,YU hai-bin,LIANG wei,Energy Effici-ency Packet Optimization Algorithm for Wireless Sensor Network.Control and Decision (2006), p.180-183.

[5] Lettieri P.,Srivastava M B.,Adaptive Frame Length Control for Improving Wireless Link Throughput, Range and Energy Efficiency. Proc of IEEE INFOCOM (1998), p.564-571.

[6] Akyildiz I F.,Pompili D.,Melodia T. Under-water acoustic sensor networks:Research challenges. Elsevier Journal of Ad Hoc Networks (2005), p.257-277.

[7] Krishnamachari B.,Estrin D.,Wicker S. Modelling datacentric routing in wireless sensor networks. Proc of IEEE Infocom, NY (2002), p.02-14. 